\title{
The Angular Momentum of Brightest Cluster Galaxies
}

\section{S. Brough ${ }^{1}$, K.-V. Tran ${ }^{2,3}$ and A. von der Linden ${ }^{4}$}

${ }^{1}$ Australian Astronomical Observatory, PO Box 915, North Ryde, NSW 1670, Australia

${ }^{2}$ George P. \& Cynthia W. Mitchell Institute for Fundamental Physics \& Astronomy, Department of Physics \& Astronomy, Texas A\&M University, College Station, TX 77843, USA

${ }^{3}$ Institute for Theoretical Physics, University of Zurich, CH 8057, Switzerland

${ }^{4}$ Kavli Institute of Particle Astrophysics \& Cosmology (KIPAC), Stanford University, 452 Lomita Mall, Stanford, CA 94305, USA

Abstract. Massive Brightest Cluster Galaxies (BCGs) are observed to have a range of angular momenta, suggesting a variety of merging histories.

Keywords. galaxies: elliptical and lenticular, cD, galaxies: kinematics and dynamics

\section{Summary}

Brightest Cluster Galaxies (BCGs) include the most massive galaxies in the Universe and are predicted to undergo more merging than less massive galaxies. However, the observational evidence for recent BCG growth via merging is contradictory (e.g. Collins et al. 2009, Lidman et al. 2012). BCGs should also have relatively low angular momentum due to their predicted rich merger histories, but this remains unclear.

We present VLT/VIMOS integral field spectroscopy of 10 BCGs at $z \sim 0.1$ from SDSS (Brough et al. 2011, Jimmy et al. in prep.). Three have companions within $20 \mathrm{kpc}$. These galaxies extend the mass range analysed in existing surveys (Fig. 1).

BCG merging activity, as indicated by angular momentum, is diverse - two BCGs (as well as the massive companions) are fast rotators, while others have very low angular momentum (Fig. 1). This may indicate that dry merging is taking place up until today.
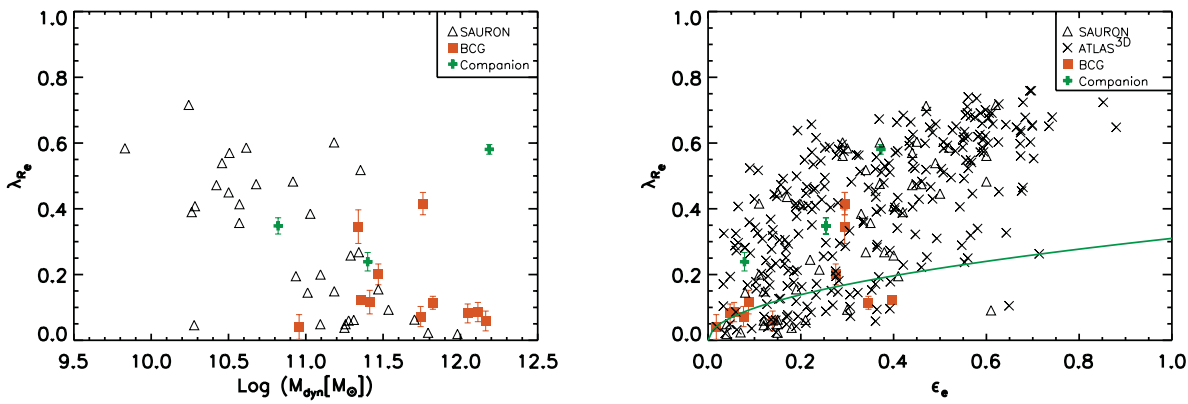

Figure 1. Left: Angular momentum parameter, $\lambda_{R e}$, versus dynamical mass. Right: $\lambda_{R e}$ versus ellipticity. Rotating galaxies lie above the green line. Some of these massive galaxies have unexpectedly high angular momentum.

\section{References}

Brough, S., et al. 2011, MNRAS, 413, 1236

Collins, C. A., et al. 2009, Nature, 458, 603

Lidman, C., et al. 2012, MNRAS, 427, 550 